\title{
Nuclear organization of cholinergic, putative catecholaminergic, serotonergic and orexinergic systems in the brain of the African pygmy mouse (Mus minutoides): Organizational complexity is preserved in small brains
}

\author{
Jean-Leigh Kruger ${ }^{1}$, Nina Patzke ${ }^{1}$, Kjell Fuxe ${ }^{2}$, Nigel C. Bennett ${ }^{3}$ \& Paul R. Manger ${ }^{1}$.
}

${ }^{1}$ School of Anatomical Sciences, Faculty of Health Sciences, University of the Witwatersrand, 7 York Road, Parktown 2193, Johannesburg, Republic of South Africa.

${ }^{2}$ Department of Neuroscience, Karolinska Institutet, Retzius väg 8, S-171 77 Stockholm, Sweden.

${ }^{3}$ Mammal Research Institute, Department of Zoology and Entomology, University of Pretoria, Pretoria 0002, South Africa.

Correspondence to: Paul Manger

\author{
School of Anatomical Sciences \\ Faculty of Health Sciences \\ University of the Witwatersrand \\ 7 York Road, Parktown, 2193 \\ Johannesburg, REPUBLIC OF SOUTH AFRICA. \\ Ph: +2711 7172497 Fax: +27 117172422 \\ Paul.Manger@wits.ac.za
}

\begin{abstract}
This study investigated the nuclear organization of four immunohistochemically identifiable neural systems (cholinergic, catecholaminergic, serotonergic and orexinergic) within the brain of the African pygmy mouse (Mus minutoides). The African pygmy mice studied had a brain mass of around $275 \mathrm{mg}$, making these the smallest rodent brains to date in which these neural systems have been investigated. In contrast to the possibility that in this small brain there would be fewer subdivisions of these neural systems, we found that all
\end{abstract}


nuclei generally observed for these systems in other rodent brains were also present in the brain of the African pygmy mouse. As with other rodents of the subfamily Murinae previously studied, we observed the presence of cortical cholinergic neurons and a compactly organized locus coeruleus. These two features of these systems have not been observed in the non-Murinae rodents studied to date. Thus, the African pygmy mouse displays what might be considered a typically Murinae brain organization, and despite its small size, the brain does not appear to be any less complexly organized than other rodent brains, even those that are over 100 times larger such as the Cape porcupine brain. Thus, the current study underscores the conserved manner in which changes in brain size affect the evolution of nuclear organization of neural systems, whereby species belonging to the same order generally have the same number and complement of the subdivisions, or nuclei, of specific neural systems despite obvious differences in brain size, phenotype or time since evolutionary divergence.

\section{Introduction}

The order Rodentia is comprised of over 2300 species in 34 families (Jansa and Weksler, 2004; Wilson and Reeder, 2005). Within the family Muridae are the true rats and mice, including the African pygmy mouse (Mus minutoides) (Wilson and Reeder, 2005). This omnivorous nocturnal mouse is one of the smallest extant African rodents with an average body mass of only 8g and inhabits both arid areas and riverine forest (Kerley, 1991; Downs and Perrin, 1996; Monadjem, 1999). M. minutoides is an interesting species, not only because of its diminutive size, but also because this species exhibits an unusual form of sex determination. A large number $(\sim 75 \%)$ of fertile females of this species have been found with the XY chromosome pairing, which would typically be associated with a male genotype (Veyrunes et al., 2010).

The current study investigates several systems within in the brain of M. minutoides, using immunohistochemical techniques to reveal, the cholinergic, putative catecholaminergic, serotonergic and orexinergic systems. The cholinergic system, which is found from the olfactory bulbs to the spinal cord, is involved in certain sleep stages and wakefulness (Jones and Cuello, 1989; Siegel, 2006), learning and memory (Reiner and Fibiger, 1995; Ferreira et al., 2001), the determination of EEG patterns such as during REM sleep (Siegel, 2006) and even in the generation of the conscious experience (Woolf and Hammeroff, 2001). The catecholaminergic system is also widely distributed throughout the brain and has a variety of 
functions including cognitive (memory, abstract thinking and motor planning) (Previc, 1999; Agnati et al., 2003), neuroendocrine (including stress and growth functions) (Fuxe, 1964; Fuxe et al., 1970; Tillet and Kitahama, 1998; Leshin et al., 1995) and cardiorespiratory functions (Feldman and Ellenberger, 1988; Fuxe et al., 1987). The serotonergic system plays a role in mood, sleep and pain regulation (Törk, 1990; Jacobs and Azmitia, 1992), and orexin (hypocretin) is a hypothalamic neurmodulatory peptide produced by neurons in the periforincal and lateral hypothalamus that has been shown to have a role in the control of feeding and arousal (Mintz et al., 2001; Ferguson and Samson, 2003; Zeitzer et al., 2003; Kirouac et al., 2005; Takakusaki et al., 2005).

If we consider the pygmy mouse's lifestyle, phenotype, sex determination, body size and most importantly its tiny absolute brain mass $(\sim 275 \mathrm{mg})$, this species becomes uniquely placed to shed further light on rodent brain evolution and more generally on patterns of brain evolution across mammals. Studies of the cholinergic, catecholaminergic, serotonergic and orexinergic systems in a range of rodent species including the greater canerat (Dwarika et al., 2008), the Highveld gerbil (Moon et al., 2007), the African molerats (Da Silva et al., 2006; Bhagwandin et al., 2006, 2008, 2011), the Cape porcupine (Limacher et al., 2008), the Syrian hamster (McGranaghan and Piggins, 2001; Mintz et al., 2001), the Siberian hamster (McGranaghan and Piggins, 2001) and the unstriped Nile grass rat (Novak and Albers, 2002), complement the wealth of published information on typical laboratory rodents (Dahlström and Fuxe, 1964; Fuxe, 1964; Fuxe et al., 1969, 1970; Hökfelt et al., 1976; 1984; Steinbusch, 1981; Skagerberg et al., 1988; Jones and Cuello, 1989, Peyron et al., 1998; Chen et al., 1999; Cutler et al., 1999; Wagner et al., 2000; Baldo et al., 2003; Espana et al., 2005; Khorooshi and Klingenspor, 2005; Kirouac et al., 2005; Vidal et al., 2005; Nixon and Smale, 2007). Thus, to date, a range of rodent species with varying life histories, phenotypes (including a range of brain and body sizes) and from a range of rodent familes have been investigated; however, a rodent species with a very small brain has yet to be examined. Apart from the demonstration of the variation found in the species belonging to the family Muridae (Dahlström and Fuxe, 1964; Björklund and Lindvall, 1984; Limacher et al., 2008 - locus coeruleus proper different in Murids compared to other rodents; Bhagwandin et al., 2006 cortical cholinergic neurons only found in Murid rodents, but not other species) all species within this order have the same nuclear organisation of these systems regardless of the variation in phenotype, life history, or evolutionary temporal distance (Manger, 2005). Thus, the present study poses the question of whether, with a very small brain, the nuclear 
organization of the systems under investigation are less complexly organized, as might be proposed from theories and observations across mammalian orders (Ebbeson, 1980; Stephan et al., 1981), or, despite the small brain, does M. minutoides display a typically rodent-like organization of these system (Manger, 2005).

\section{Materials and Methods}

The brains from three adult male African pygmy mice were used in this study (average body mass $=8.8 \mathrm{~g}$; average brain mass $=275 \mathrm{mg}$ ). All the mice were captured from a wild population in the Eastern Cape of South Africa and were treated and used according to the guidelines of the University of the Witwatersrand Animal Ethics Committee. The animals were euthanased (Euthanase, $200 \mathrm{mg}$ sodium pentobarbital $/ \mathrm{kg}$, i.p.) and upon cessation of respiration, perfused intracardially with an initial rinse of $0.9 \%$ saline solution at $4^{\circ} \mathrm{C}(10 \mathrm{ml})$, which was followed by a solution of $4 \%$ paraformaldehyde in $0.1 \mathrm{M}$ phosphate buffer (PB) $(10 \mathrm{ml})$. After removal from the skull, each brain was post-fixed overnight in the paraformaldehyde solution and subsequently stored in anti-freeze at $-20^{\circ} \mathrm{C}$. Before sectioning, the brains were allowed to equilibrate in $30 \%$ sucrose in $0.1 \mathrm{M} \mathrm{PB}$ at $4^{\circ} \mathrm{C}$. Each brain was then frozen and sectioned into $50 \mu \mathrm{m}$ thick serial coronal sections. A one in six series of sections was used for Nissl, myelin, choline-acyltransferase (ChAT), tyrosine hydroxylase (TH), serotonin (5HT) and orexin (hypocretin/OxA). Sections for Nissl staining were first mounted on $0.5 \%$ gelatine coated slides, cleared in a solution of $1: 1$ absolute alcohol and chloroform and then stained with $1 \%$ cresyl violet. The myelin series sections were refrigerated for two weeks in 5\% formalin then mounted on $1 \%$ gelatine coated slides and stained with a modified silver stain (Gallyas, 1979).

The sections for immunohistochemical staining were treated for $30 \mathrm{~min}$ in an endogenous peroxidise inhibitor (49.2\% methanol: $49.2 \% 0.1 \mathrm{M} \mathrm{PB}: 1.6 \%$ of $30 \%$ hydrogen peroxide) followed by three $10 \mathrm{~min}$ rinses in $0.1 \mathrm{M}$ PB. Sections were then preincubated for 2 $\mathrm{h}$, at room temperature, in blocking buffer ( $3 \%$ normal goat serum, NGS, for TH, serotonin and orexin sections; $3 \%$ normal rabbit serum, NRS, for ChAT sections, $2 \%$ bovine serum albumin for all sections and $0.25 \%$ Triton- $\mathrm{X}$ in $0.1 \mathrm{M}$ PB for all sections). Thereafter sections were incubated in the primary antibody solution in blocking buffer for $48 \mathrm{~h}$ at $4^{\circ} \mathrm{C}$. Anticholineacetyltransferase (AB144P, Millipore, raised in goat) at a dilution of 1:3000 was used to reveal cholinergic neurons. Anti-tyrosine hydroxylase (AB151, Millipore, raised in rabbit) at a dilution of 1:7500 revealed the putative catecholaminergic neurons. Serotonergic neurons 
were revealed using anti-serotonin (AB938, Millipore, raised in rabbit) at a dilution of 1:10000. Orexinergic neurons were revealed using anti-OrexinA (AB3704, Millipore, raised in rabbit) at a dilution of 1:3000. This incubation was followed by three $10 \mathrm{~min}$ rinses in $0.1 \mathrm{M} P B$ and the sections were then incubated in a secondary antibody solution (1:7500 dilution of biotinylated anti-rabbit $\mathrm{IgG}$ for $\mathrm{TH}$, serotonin and orexin sections, or a 1:750 dilution of biotinylated anti-goat IgG for ChAT sections, in a blocking buffer containing 3\% NGS/NRS and $2 \% \mathrm{BSA}$ in $0.1 \mathrm{M} \mathrm{PB}$ ) for $2 \mathrm{~h}$ at room temperature. This was followed by three 10 min rinses in $0.1 \mathrm{M} \mathrm{PB}$, after which sections were incubated for $1 \mathrm{~h}$ in $\mathrm{AB}$ solution (Vector Labs), followed by three $10 \mathrm{~min}$ rinses in $0.1 \mathrm{M}$ PB. Sections were then placed in a $0.05 \%$ diaminobenzidine $(\mathrm{DAB})$ in $0.1 \mathrm{M}$ PB solution for $5 \mathrm{~min}$, followed by the addition of $3 \mu \mathrm{l}$ of $3 \%$ hydrogen peroxide to each $1 \mathrm{ml}$ of solution in which each section was immersed.

Chromatic precipitation was visually monitored and verified under a low power stereomicroscope. Staining continued until such time as the background stain was at a level that would assist reconstruction without obscuring the immunopositive neurons. Precipitation was arrested by placing sections in $0.1 \mathrm{M} \mathrm{PB}$, followed by two more rinses in this solution. Sections were then mounted on $0.5 \%$ gelatine coated glass slides, dried overnight, dehydrated in a graded series of alcohols, cleared in xylene and coverslipped with Depex. The controls employed in this experiment included the omission of the primary antibody and the omission of the secondary antibody in selected sections for which no staining was evident.

Sections were examined under low power steromicroscope and using a camera lucida, the architectonic borders of the sections were traced following the Nissl and myelin stained sections. Sections containing the immunopositive neurons were matched to the drawings and the neurons were marked. All drawings were then scanned and redrawn using the Canvas 8 drawing program. All architectonic nomenclature was taken from the atlas of a rodent brain (Paxinos and Watson, 2009). The nomenclature used for the cholinergic system was adopted from Woolf (1991), Limacher et al. (2008) and Bhagwandin et al. (2008), the catecholaminergic from Hökfelt et al. (1984), Smeets and Gonzalez (2000), Limacher et al. (2008) and Bhagwandin et al. (2008), the serotonergic from Törk (1990), Limacher et al. (2008) and Bhagwandin et al. (2008) and the orexinergic from Kruger et al. (2010a), Bhagwandin et al. (2011) and Gravett et al (2011). While we use the standard nomenclature for the catecholaminergic system in this paper, we realise that the neuronal groups we revealed with tyrosine hydroxylase immunohistochemistry may not correspond directly with these nuclei as has been described in previous studies by Dahlström and Fuxe (1964), Hökfelt 
et al. (1976), Meister et al. (1988), Kitahama et al. (1990, 1996), and Ruggiero et al. (1992).

However, given the striking similarity of the results of the tyrosine hydroxylase

immunohistochemistry to that seen in other mammals we feel this terminology is appropriate.

Clearly further studies in the mole-rat species used with a wider range of antibodies, such as those to phenylethanolamine-N-methyltransferase (PNMT), dopamine-b-hydroxylase (DBH) and aromatic L-amino acid decarboxylase (AADC) would be required to fully determine the implied homologies ascribed in this study. We address this potential problem with the caveat of putative catecholaminergic neurons where appropriate in the text.

\section{Abbreviations:}

III - oculomotor nucleus

IV - trochlear nucleus

Vmot - motor division of trigeminal nerve nucleus

Vsens - sensory division of trigeminal nerve nucleus

$\mathrm{VI}$ - abducens nucleus

VIId - dorsal division of facial nerve nucleus

VIIv - ventral division of facial nerve nucleus

$\mathrm{X}$ - dorsal motor vagus nucleus

XII - hypoglossal nucleus

$3 \mathrm{~V}-$ third ventricle

$4 \mathrm{~V}$ - fourth ventricle

$7 \mathrm{n}-$ facial nerve

A1 - caudal ventrolateral medullary tegmental nucleus

A2 - caudal dorsomedial medullary nucleus

A4 - dorsal medial division of locus coeruleus

A5 - fifth arcuate nucleus

A6c - compact portion of locus coeruleus

A7d - nucleus subcoeruleus, diffuse portion

A7sc - nucleus subcoeruleus, compact portion

A8 - retrorubral nucleus 
A91 - substantia nigra, lateral

A9m - substantia nigra, medial

A9pc - substantia nigra, pars compacta

$\mathrm{A} 9 \mathrm{v}$ - substantia nigra, ventral, pars reticulata

A10 - ventral tegmental area

A10c - ventral tegmental area, central

A10d - ventral tegmental area, dorsal

A10dc - ventral tegmental area, dorsal caudal

A11 - caudal diencephalic group

A12 - tuberal cell group

A13 - zona incerta cell group

A14 - rostral periventricular nucleus

A15d - anterior hypothalamic group, dorsal division

A15v - anterior hypothalamic group, ventral division

A16 - catecholaminergic neurosn of the olfactory bulb

ac - anterior commissure

Amyg - amygdala

$\mathrm{AP}$ - area postrema

$\mathrm{ARC}$ - arcuate nucleus of the hypothalamus

B9 - supralemniscal serotonergic nucleus

$\mathrm{C} 1$ - rostral ventrolateral medullary tegmental group

$\mathrm{C} 2$ - rostral dorsomedial medullary nucleus

$\mathrm{C} 3$ - rostral dorsal midline medullary nucleus

$\mathrm{C}$ - caudate nucleus

ca - cerebral aqueduct

cc - corpus callosum

Cer - cerebellum

CLi - caudal linear nucleus

$\mathrm{CN}$ - deep cerebellar nucleus 
CVL - caudal ventrolateral serotonergic group

Diag.B - diagonal band of Broca

DRc - dorsal raphe, caudal division

DRd - dorsal raphe, dorsal division

DRif - dorsal raphe, interfascicular division

DR1 - dorsal raphe, lateral division

DRp - dorsal raphe, peripheral division

$\mathrm{DRv}$ - dorsal raphe, ventral division

DT - dorsal thalamus

EW - Edinger-Westphal nucleus

$f-$ fornix

$\mathrm{fr}-$ fasciculus retroflexus

$\mathrm{GC}$ - central grey matter

GCL - granular cell layer of olfactory bulb

GP - globus pallidus

$\mathrm{Hbl}$ - lateral habenular nucleus

$\mathrm{Hbm}$ - medial habenular nucleus

HIP - hippocampus

Hyp - hypothalamus

Hyp.d - dorsal hypothalamic cholinergic nucleus

Hyp.1 - lateral hypothalamic cholinergic nucleus

Hyp.v - ventral hypothalamic cholinergic nucleus

$\mathrm{IC}$ - inferior colliculus

ic - internal capsule

io - inferior olive

IP - interpeduncular nucleus

Is.Call/TOL - islands of Calleja/olfactory tubercle

LDT - laterodorsal tegmental nucleus

LGv - ventral lateral geniculate nucleus 
LOT - lateral olfactory tract

LV - lateral ventricle

$\mathrm{Mc}$ - orexinergic main cluster

mcp - middle cerebellar peduncle

$\mathrm{MnR}$ - median raphe nucleus

$\mathrm{mtf}$ - medullary tegmental field

N.Acc - nucleus accumbens

N.Amb - nucleus ambiguus

N.Bas - nucleus basalis

Neo - neocortex

OB - olfactory bulb

OC - optic chiasm

OT - optic tract

Otc - orexinergic optic tract cluster

$\mathrm{P}$ - putamen nucleus

PB - parabrachial nucleus

$\mathrm{PBg}$ - parabigeminal nucleus

$\mathrm{PC}$ - cerebral peduncle

PIR - piriform cortex

PPT - pedunculopontine nucleus

Pta - pretectal area

py - pyramidal tract

$\mathrm{pVII}$ - preganglionic motor neurons of the superior salivatory nucleus or facial nerve

pIX - preganglionic motor neurons of the inferior salivatory nucleus

$\mathrm{R}$ - reticular nucleus of the thalamus

$\mathrm{RMg}$ - raphe magnus nucleus

$\mathrm{ROb}$ - raphe obscurus nucleus

$\mathrm{RPa}$ - raphe pallidus nucleus

$\mathrm{RVL}$ - rostral ventrolateral serotonergic group 
S - septal nuclear complex

$\mathrm{SC}$ - superior colliculus

scp - superior cerebellar peduncle

Sep.M - medial septal nucleus

$\mathrm{SON}$ - superior olivary nucleus

sp5 - spinal trigeminal tract

$\mathrm{VCO}$ - ventral cochlear nucleus

VPO - ventral pontine nucleus

xpy - decussation of the pyramidal tract

xscp - decussation of the superior cerebellar peduncle

zi - zona incerta

Zic - orexinergic zona incerta cluster

\section{Results}

The current study delineates the nuclear organization of the cholinergic, putative catecholaminergic, serotonergic and orexinergic neural systems of the African pygmy mouse (Fig. 1). The three pygmy mice used in this study had an average body mass of $8.8 \mathrm{~g}$ and an average brain mass of $275 \mathrm{mg}$, making the African pygmy mouse brain the smallest of any rodent species studied to date; however, this diminutively sized brain (in comparison to the mass of previously studied rodent brains) has not led to any reduction in the number of complement of nuclei present. In fact, the overall complexity of neural systems investigated herein within the pygmy mouse brain show all the typical subdivisions, across all brain regions, that would be expected in a rodent brain (e.g. Bhagwandin et al., 2008, 2011; Limacher et al., 2008).

\subsection{Cholinergic nuclei}

The cholinergic system can be subdivided into the cortical interneurons, the striatal, basal forebrain, diencephalic, pontomesencephalic and the cranial motor nerve nuclei (Woolf, 1991), each subdivision containing a cluster of distinct nuclei (Figs. 1,2).

Cholineacetyltransferase immunoreactive neurons $(\mathrm{ChAT}+)$ were identified in all these subdivisions, including the cortical cholinergic interneurons, which have a variable 
occurrence across rodent species studied to date (e.g. Bhagwandin et al., 2006).

\subsubsection{Cortical cholinergic neurons}

In a previous study (Bhagwandin et al., 2006) it was demonstrated that the appearance of cholinergic interneurons within the cerebral cortex of rodents appears to be limited to the members of the Murid family. The African pygmy mouse (Mus minutoides) is a member of this family, and in the current study we observed cortical neurons immunoreactive to the ChAT antibody through all regions of the cortex (Fig. 2B). The majority of these neurons were found in the supragranular cortical layers, but were observed to occur in all cortical layers except layer one. The ChAT + neurons were bipolar in nature with the majority being vertically oriented within the cortical mantle.

\subsubsection{Striatal cholinergic interneurons}

ChAT + neurons were found in the caudate/putamen complex, the globus pallidus, the nucleus accumbens, the Islands of Calleja and the olfactory tubercle (Fig. 1C-H). A moderate density of ChAT + neurons were found within the caudate/putamen and throughout the globus pallidus, but most densely at its borders with the putamen and nucleus basalis (Fig. 2A,C). Through the nucleus accumbens a moderate density of ChAT + neurons was observed, and at the ventral border of this nucleus, they appear to intermingle with the most dorsal cholinergic neurons of the olfactory tubercle (Fig. 2A). The ChAT+ neurons within the olfactory tubercle and Islands of Calleja were found in the most ventral portion of the anterior telencephalon (Fig. 2A). Throughout the olfactory tubercle a moderate density of ChAT + neurons were observed, and within the most ventral portion of this region clusters of $\mathrm{ChAT}+$ neurons were observed to form the Islands of Calleja.

\subsubsection{Cholinergic nuclei of the basal forebrain}

Cholinergic nuclei that could be identified within the basal forebrain of the African pygmy mouse included the medial septal nucleus, the Diagonal band of Broca and the nucleus basalis (Fig. 1D-G). The medial septal nucleus exhibited a moderate to high density of ChAT+ neurons and was located within the rostral half of the medial wall of the septal nuclear complex immediately below the rostrum of the corpus callosum. The ChAT + neurons forming the diagonal band of Broca were evidenced as a high density of neurons located in the ventromedial corner of the cerebral hemisphere anterior to the hypothalamus 
(Fig. 2A). It was possible to divide this nucleus into both horizontal and vertical limbs, but this was not deemed necessary since it would not add any value to the description. A cluster of moderate to high-density ChAT + neurons located anterior and ventral to the globus pallidus and caudal to the olfactory tubercle were assigned to the nucleus basalis (Fig. 2C). At the posterior portion of this nucleus, the neurons appear to be continuous with those of the globus pallidus.

\subsubsection{Diencephalic cholinergic nuclei}

ChAT + neurons were found in the medial habenular nucleus, as well as the dorsal, lateral and ventral hypothalamic clusters (Fig. 1F-I). The medial habenular nucleus was located in the dorsomedial aspect of the diencephalon adjacent to the third ventricle. The ChAT + neurons within this nucleus were very densely packed (Fig. 2D). The three clusters of ChAT + neurons within the hypothalamus all showed moderate to weak immunoreactivity, but were clearly observed. The dorsal cluster was found in the dorsomedial aspect of the hypothalamus between the third ventricle and fornix (Fig. 2C), the lateral cluster was found in the dorsolateral aspect of the hypothalamus, lateral to the fornix, while the ventral cluster was located in the ventral medial portion of the hypothalamus were adjacent to the neurons of the A12 nucleus (see below).

\subsubsection{Pontomesencephalic cholinergic nuclei}

ChAT + immunoreactive neurons were used to delineate the parabigeminal nucleus, as well as the pedunculopontine (PPT) and the laterodorsal (LDT) nuclei (Fig. 1K-M). The parabigeminal nucleus was located at the very lateral margin of the pontine tegementum in a location ventral the inferior colliculus. Within this nucleus the ChAT+ neurons were moderately densely packed (Fig. 1E). The ChAT+ neurons forming the PPT were located within the dorsal aspect of the pontine tegementum surrounding the superior cerebellar peduncle. A moderate to high density of ChAT+ neurons were observed in this region (Fig.. E,F). Within the periventricular grey matter, caudal to the oculomotor nucleus, a moderate to high density of ChAT + neurons were designated as those forming the LDT nucleus (Fig. 2F). The ventrolateral border of the LDT neurons was contiguous with the dorsomedial border of the PPT nucleus, the only reason to separate these two nuclei being the transition from the periventricular grey matter to the pontine tegementum. 


\subsubsection{Cholinergic cranial nerve motor nuclei}

These nuclei were found in positions typical of all mammals (Woolf, 1991; Manger et al., 2002a; Maseko et al., 2007). The ChAT+ nuclei identified in the African pygmy mouse include: the oculomotor (III), trochlear (IV), motor division of the trigeminal (Vmot), adbucens (VI), dorsal and ventral subdivisions of the facial (VIId and VIIv), nucleus ambiguus, dorsal motor vagus (X), hypoglossal (XII), Edinger-Westphal (EW), medullary tegemental field (mtf) and the preganglionic motor neurons of the salivatory (pVII) and the glossopharyngeal (pIX) nerves (Fig. 1J-R).

\subsection{Putative catecholaminergic nuclei}

Tyrosine hydroxylase $(\mathrm{TH})$ immunoreactivity was used to reveal the putatively catecholaminergic neurons (see above) in the African pygmy mouse brain. The nuclei formed by these neurons were arranged in a number of identifiable nuclear complexes that extended from the olfactory bulb through to the spinomedullary junction (Fig. 1). These complexes were identical to that seen in other rodents and could be divided into the olfactory bulb, diencephalic, midbrain, pontine and medullary nuclear clusters. For simplicity, in the current description, the nuclei are referred to using the nomenclature of Dahlström and Fuxe (1964) and Hökfelt et al. (1984). No putatively catecholaminergic nuclei outside the classically defined nuclei (e.g. Smeet and Gonzalez, 2000) were observed.

\subsubsection{Olfactory bulb (A16)}

A high density of $\mathrm{TH}+$ neurons were found in and around the stratum granulosum of the olfactory bulb. These neurons probably represent the periglomerular dopaminergic interneurons, were small in size, and were found in equal density surrounding the entire glomeruli (Fig. 1A,B).

\subsubsection{Diencephalic nuclei (A15-A11)}

Six clusters of TH+ neurons, forming distinct nuclei, were observed within the hypothalamus, these being: the anterior hypothalamic group, dorsal division (A15d); the anterior hypothalamic group, ventral division $(\mathrm{A} 15 \mathrm{v})$; the rostral periventricular cell group (A14); the zona incerta (A13); the tuberal cell group (A12); and the caudal diencephalic group (A11) (Fig. 1E-J). Within the dorsal anterior portion of the hypothalamus, between the third ventricle and the fornix, a moderate density of $\mathrm{TH}+$ neurons was designated as the $\mathrm{A} 15 \mathrm{~d}$ 
nucleus (Fig. 3A). The $\mathrm{TH}+$ neurons forming the A15v nucleus were located in the ventrolateral portion of the hypothalamus in a moderate density close to the floor of the brain. A low to moderate density of $\mathrm{TH}+$ neurons within the hypothalamus, forming two columns adjacent to the lateral walls of the third ventricle, were assigned as the A14 nucleus (Fig. 3A,B). Within the dorsolateral aspect of the hypothalamus, lateral to the fornix and intermingling with the neurons forming the zona incerta of the ventral thalamus, was a low to moderate density of $\mathrm{TH}+$ neurons belonging to the A13 nucleus (Fig. 3B). $\mathrm{TH}+$ neurons assigned to the A12 nucleus were located in the ventromedial portion of the hypothalamus, surrounding and below the floor of the third ventricle in the vicinity of the arcuate nucleus (Fig. 3B). Lastly, within the hypothalamic grey matter adjacent to the posterior pole of the third ventricle, a moderate density of TH+ neurons formed the A11 nucleus.

\subsubsection{Midbrain nuclei $(A 10-A 8)$}

Within the midbrain, the $\mathrm{TH}+$ neurons were found within the ventral tegmental area (the A10 complex, including the A10, A10c, A10d, A10dc nuclei), the substantia nigra (the A9 complex, including the A9pc, A91, A9v, A9m nuclei) and the retrorubral nucleus (A8) within the midbrain tegmentum (Fig. 1J,K). The nuclei of the A10 complex extended from within the periaqueductal grey matter around the base of the aqueduct, into the tegmentum below the periaqueductal grey matter around the midline, through to and around the interpeduncular nucleus. A high density of $\mathrm{TH}+$ neurons, found dorsal and dorsolateral to the interpeduncular nucleus, between this nucleus and the root of the oculomotor nerve (IIIn), was assigned to the A10 nucleus. Immediately dorsal to the interpeduncular nucleus, in a location just anterior to the decussation of the superior cerebellar peduncle, was a dense cluster of $\mathrm{TH}+$ neurons forming the A10c nucleus. Immediately dorsal to A10c, between it and the oculomotor nucleus, was a dense bilateral parasagittal cluster of $\mathrm{TH}+$ neurons that formed the A10d nucleus (Fig. 3C). The TH+ neurons assigned to the A10dc nuclear complex were found within the periaqueductal grey matter adjacent to and surrounding the ventral half of the cerebral aqueduct.

The substantia nigra nuclear complex was located in the ventral and lateral portions of the midbrain tegmentum, lying just dorsal to the cerebral peduncles. Evidence for four distinct nuclei namely, the substantia nigra, pars compacta (A9pc), substantia nigra, ventral or pars reticulata (A9v), substantia nigra, pars lateralis (A9l) and substantia nigra, pars medialis (A9m), were found within the A9 complex. A9pc was seen to be a dense band of TH+ 
neurons that ran from medial to lateral immediately dorsal to the cerebral peduncle. Throughout the cerebral peduncle ventral to A9pc, several $\mathrm{TH}+$ neurons were assigned to the A9v nucleus (Fig. 3C). At the lateral edge of A9pc, a moderate density and loose aggregation of $\mathrm{TH}+$ neurons formed the A9l nucleus. Medial to A9pc and lateral to the root of the oculomotor nerve (IIIn), a dense cluster of $\mathrm{TH}+$ neurons formed the A9m nucleus. Scattered throughout the midbrain tegmentum, in a position caudal to the magnocellular division of the red nucleus and dorsal to the A9 complex, was a sparsely packed but relatively numerous, cluster of $\mathrm{TH}+$ neurons that formed the $\mathrm{A} 8$ nucleus (Fig. 3C).

\subsubsection{Rostral rhombencephalon - the locus coeruleus complex, A7 - A4}

Within the pontine region a large number of $\mathrm{TH}+$ neurons forming the locus coeruleus complex were readily observed (Fig. 1M-O). The locus coeruleus complex could be readily subdivided into five nuclei, these being: the subcoeruleus compact portion $(\mathrm{A} 7 \mathrm{sc})$, subcoeruleus diffuse portion (A7d), locus coeruleus compact portion (A6c), fifth arcuate nucleus (A5), and the dorsal medial division of locus coeruleus (A4). Within the dorsal portion of the pontine tegmentum adjacent to the ventrolateral region of the periaqueductal grey matter, a tightly packed cluster of $\mathrm{TH}+$ neurons represented the $\mathrm{A} 7$ compact portion of the LC. This division is the same as what was previously described as the subcoeruleus (Dahlström and Fuxe, 1964; Olson and Fuxe, 1972). Ventral and lateral to the A7sc, a diffusely organised aggregation of $\mathrm{TH}+$ neurons formed the $\mathrm{A} 7 \mathrm{~d}$ nuclear complex. These neurons are located both medially and laterally around the trigeminal motor nucleus (Vmot). Within the lateral portion of the periventricular grey matter a tightly packed, moderate to high density of $\mathrm{TH}+$ neurons were assigned to the A6c nucleus (Fig. 3C). The neurons of this group were found adjacent to the wall of the fourth ventricle, stretching across the periventricular grey matter to its lateral edge as seen in the laboratory rat (Dahlström and Fuxe, 1964), and were not seen as a loosely packed cluster of neurons located in the ventrolateral half of the periventricular grey matter as seen in other non-Murid rodent species (Moon et al., 2007; Dwarika et al., 2008; Bhagwandin et al., 2008; Limacher et al., 2008). In the ventrolateral pontine tegmentum lateral to the superior olivary nucleus and lateral to Vmot and A7d, a small cluster of TH+ neurons formed the A5 nucleus. These neurons formed a rough meshlike dendritic network around the ascending fascicles located within the ventrolateral pontine tegmentum. Immediately adjacent to the wall of the fourth ventricle, in the dorsolateral portion of the periaqueductal grey matter, a very $\mathrm{TH}+$ neurons represent the 
A4 nucleus.

\subsubsection{Medullary nuclei (C1, C2, C3, A1, A2, Area postrema)}

Within the medulla of the African pygmy mouse we found evidence for six putative catecholaminergic nuclei these being: rostral ventrolateral tegmental group $(\mathrm{C} 1)$, rostral dorsomedial group (C2), rostral dorsal midline group (C3), caudal ventrolateral tegmental group (A1), caudal dorsomedial group (A2) and area postrema (AP) (Fig. 1N-R). A low density of $\mathrm{TH}+$ neurons forming the $\mathrm{C} 1$ nucleus were found in the ventrolateral medulla from the level of the facial nerve nucleus to the mid-level of nucleus ambiguus. Continuing in the ventrolateral medulla, a column of $\mathrm{TH}+$ neurons located laterally to the posterior most part of the $\mathrm{C} 1$ nucleus and extending to the spinomedullary junction, was designated as forming the A1 nucleus. The A1 column was distinguished from the ventrolateral C1 column by occupying a position lateral to the lateral reticular nucleus and nucleus ambiguus, whereas $\mathrm{C} 1$ was located medial to these structures. In the dorsal part of the medulla, in the region of the anterior part of the dorsal and medial border of the nucleus tractus solitarius, a distinct cluster of numerous $\mathrm{TH}+$ neurons was designated as the $\mathrm{C} 2$ nucleus. Within this nucleus there was a clear region close to the floor of the fourth ventricle termed the dorsal strip and a continuation of this cluster into the region of the tractus solitarius termed the rostral subdivision of the $\mathrm{C} 2$ nucleus. Within the dorsal medial medullary tegmentum at the midline, dorsal to the raphe obscurus and close to the floor of the fourth ventricle, a very few $\mathrm{TH}+$ neurons representing the $\mathrm{C} 3$ nucleus were found. Between the caudal portions of the dorsal motor vagus and hypoglossal cranial nerve nuclei, a small number of TH+ neurons represented the A2 nucleus. Some of these A2 neurons were located a small distance into the dorsal caudal medullary tegmentum. Straddling the midline, dorsal to the central canal and the dorsal motor vagus nucleus, and between the most caudal region of the bilateral $\mathrm{C} 2$ nucleus, was a single large, densely packed, cluster of intensely stained $\mathrm{TH}+$ neurons, the area postrema.

\subsection{Serotonergic nuclei}

The serotonergic $(5 \mathrm{HT}+)$ nuclei identified in the African pyrgmy mouse were the same as those previously identified in all rodents and other eutherian mammals studied to date (Steinbusch, 1981; Maseko et al., 2007; Dell et al., 2010). The serotonergic nuclei were all located within the brainstem and can be divided into a rostral and a caudal cluster. Both of these clusters contained a number of distinct nuclei that are found throughout the brainstem 
from the level of the decussation of the superior cerebellar peduncle through to the spinomedullary junction (Fig. 1K-R).

\subsubsection{Rostral cluster}

Within the rostral cluster we found evidence for the caudal linear nucleus (CLi), the supralemniscal nucleus (B9), the median raphe nucleus (MnR) and the dorsal raphe complex formed of six distinct nuclei (Fig. 1K-M). The CLi nucleus was the most rostral of the serotonergic nuclei found and the $5 \mathrm{HT}+$ neurons formed a moderate density cluster around the midline immediately dorsal to the interpeduncular nucleus in a location just anterior to the decussation of the superior cerebellar peduncle (Fig. 4A). The neurons forming the B9 nucleus appeared to be a lateral extension of the neuronal cluster comprising the most ventral portion of CLi. The 5HT+ B9 neurons were found in a low to moderate density immediately caudal to the A9pc (see above) above the cerebral peduncle and extended as an arc of neurons into the lateral and ventrolateral portion of the midbrain tegmentum (Fig. 4A). The median raphe nucleus $(\mathrm{MnR})$ was characterised by two distinct, densely packed $5 \mathrm{HT}+$ neuronal columns on either side of the midline in a para-raphe position (Fig. 4B,C). The rostral border of this nucleus was coincident with the level of the decussation of the superior cerebellar peduncle and the caudal border of this nucleus was found at the level of the trigeminal motor nucleus.

Within the $5 \mathrm{HT}+$ neuronal region designated as the dorsal raphe nuclear complex there were six distinct nuclei, these being: the dorsal raphe interfascicular (DRif) nucleus, dorsal raphe ventral (DRv) nucleus, dorsal raphe dorsal (DRd) nucleus, dorsal raphe lateral (DRl) nucleus, dorsal raphe peripheral (DRp) nucleus and the dorsal raphe caudal (DRc) nucleus (Figs. 1K-M, 4B, 4C). These six nuclei were found, for the most part, within the periaqueductal and periventricular grey matter from the level of the oculomotor nucleus to the trigeminal motor nucleus. The DRif was located between the two medial longitudinal fasciculi and exhibited a high density of 5HT+ neurons. The DRv was found immediately dorsal to the DRif between and just caudal to the oculomotor nuclei. The DRv exhibited a high density of 5HT+ neurons. Immediately dorsal to DRv and ventral to the inferior border of the cerebral aqueduct a high-density cluster of 5HT+ neurons was designated as the DRd nucleus. A moderate density of 5HT+ neurons representing the DRp, were located in the ventrolateral portion of the periaqueductal grey matter lateral to the DRd and DRv. Some neurons of the DRp were found in the adjacent tegmentum and are the only ones found 
outside the periaqueductal grey matter. The $5 \mathrm{HT}+$ neurons of the DRl were located dorsolateral to the DRd and adjacent to the ventrolateral edges of the cerebral aqueduct in a low to moderate density. The neurons of this nucleus were readily distinguishable from the remainder of the dorsal raphe nuclei since they were substantially larger. As we followed the DRl caudally, where the cerebral aqueduct opened into the fourth ventricle and the DRd, DRv and DRif dissappeared, the neurons of the DR1 formed an arc across the midline of the dorsal portion of the periventricular grey matter. This caudal arc of the DRl was classified as the DRc nucleus. We classified this as an independent nucleus due to the lack of 5HT+ neurons in this region in the brain of monotremes (Manger et al., 2002c; Maseko et al., 2007; Dell et al., 2010).

\subsubsection{Caudal cluster}

Within the caudal cluster we found evidence for the raphe magnus ( $\mathrm{RMg})$, rostral and caudal ventrolateral (RVL and CVL), raphe pallidus (RPa) and raphe obscurus $(\mathrm{ROb})$ nuclei (Figs. 1M-R, 4D). This RMg was seen to be two columns of loosely aggregated moderate to large $5 \mathrm{HT}+$ neurons located either side of the midline from the level of the caudal pole of the trigeminal motor nucleus to the anterior pole of nucleus ambiguus. Within the left and right ventrolateral medullary tegmentum a distinct anteroposterior column of 5HT+ neurons extending from the level of the facial nucleus to the spinomedullary junction were observed. These have previously been termed the rostral and caudal ventrolateral serotonergic columns (e.g. Maseko et al., 2007; Moon et al., 2007; Dwarika et al., 2008). The RVL began as a lateroventral continuation of 5HT+ neurons from the lower portion of the $\mathrm{RMg}$ extending over the pyramidal tracts and consolidating as a distinct column lateral to the inferior olives. The inferior olive topologically distinguishes left and right RVL, and at the level of nucleus ambiguus the RVL becomes the CVL. The CVL continues in the caudal ventrolateral medullary tegmentum until the spinomedullary junction is reached. The number of neurons within this RVL/CVL column steadily decreases from rostral to caudal. Although the RVL and CVL are continuous in the African pygmy mice studied, and indeed several other eutherian mammals previously studied (e.g. Maseko et al., 2007; Moon et al., 2007; Dwarika et al., 2008), we make the distinction of two components of these ventrolateral columns, as the caudal portions have not been reported in the opossum or the monotremes (Crutcher and Humbertson, 1978; Manger et al., 2002c). The 5HT+ neurons forming the PRa nucleus were found in the ventral midline of the medulla associated with the pyramidal tracts. These 
neurons were for the most part located between the two pyramidal tracts, but some neurons belonging to this nucleus (based on neuronal morphology) were identified dorsal to the pyramidal tract, between it and the inferior olive. Two loosely arranged bilateral columns of $5 \mathrm{HT}+$ neurons located either side of the midline from the level of nucleus ambiguus to the spinomedullary junction were classified as the ROb. Occasional 5HT+ neurons associated with this nucleus were identified within a short distance (less than $200 \mathrm{~mm}$ ) lateral to the central columns.

\subsection{Orexinergic (hypocretinergic) nuclei}

The vast majority of orexin-A immunopositive neurons $(\mathrm{OrX}+)$ identified in the brain of the African pygmy mouse were localised in the hypothalamus (Fig. 1F-I). Within this aggregation of OrX+ cells we could identify three distinct cluster - a main cluster (Mc), a zona incerta cluster (Zic) and an optic tract cluster (Otc) (Fig. 5). The main cluster (Mc) was identified as a large group of densely packed OrX + neuronal cell bodies located lateral to the third ventricle in the perifornical region, with a moderate number of neuronal cell bodies extending medially from this area into the dorsomedial hypothalamus and a larger number extending into the lateral hypothalamic areas. From the main cluster a group of OrX+ neuronal cell bodies extended laterally into the region of the zona incerta. This cluster had a very low density of OrX + neurons that were mixed with neurons of the lateral hypothalamic cholinergic nucleus and the A13 nucleus of the catecholaminergic system (see above). The third cluster, the optic tract cluster (Otc) extended ventrolaterally from the main cluster to the ventrolateral region of the hypothalamus adjacent to the optic tract. This cluster exhibited a moderate density of $\mathrm{OrX}+$ neuronal cell bodies.

\section{Discussion}

The African pygmy mouse, with its diminutive body mass $(\sim 8 \mathrm{~g})$ and more importantly its small absolute brain mass ( $\sim 275 \mathrm{mg}$ ), can shed much light on the proposal that changes in brain size lead to increased or decreased differentiation within neural systems (Ebbesson, 1980; Stephan et al., 1981). The current study highlights that within the cholinergic, catecholaminergic, and serotonergic systems there was no difference in the number and complement of nuclei when compared with previous findings for the Cape porcupine (Limacher et al., 2008), which has a brain mass of approximately $37 \mathrm{~g}$, or around 
135 times larger that that of the pygmy mouse. Furthermore, the current findings unscore that even following major changes in phenotype, such as observed in the microphthalmic African mole rats (Bhagwandin et al., 2008, 2011), or in the current study the small body mass of the African pygmy mouse, yet again, no changes in the complement or number of nuclei are apparent as long as the comparison is limited to rodent species. Thirdly, the range of rodent species in which these systems have now been investigated (e.g. Dahlström and Fuxe, 1964; Hökfelt et al., 1976; Steinbusch, 1981; Jones and Cuello, 1989; Peyron et al., 1998; Chen et al., 1999; Wagner et al., 2000; McGranaghan and Piggins, 2001; Mintz et al., 2001; Novak and Albers, 2002; Da Silva et al., 2006; Bhagwandin et al., 2006, 2008, 2011; Nixon and Smale, 2007; Moon et al., 2007; Dwarika et al., 2008; Limacher et al., 2008), also indicate that, for the most part, it doesn't appear to matter how long a species has been separated from another of the same order (the time since they last shared a common ancestor), they will in all likelihood show the same complement and number of any given neural system (Manger, 2005). This finding appears not only to be relevant to the rodents, but appears to be more generally relevant to all mammalian orders (e.g. Maseko et al., 2007; Dell et al., 2010) and perhaps even in other vertebrate classes (Rodrigues et al., 2008). Despite this general applicability of the conserved nature of systems level brain evolution, the current study highlighted two features of interest in the brain of the African pygmy mouse regarding intraordinal brain evolution and phylogeny, these being: (1) The presence of cortical cholinergic neurons; and (2) the anatomical appearance of the locus coeruleus (A6c); both features appearing to be found only within the subfamily Murinae from the range of rodent species studied to date.

\subsection{Cortical cholinergic neurons}

Cholinergic, or ChAT immunoreactive neurons, as mentioned above, have been observed in the brains of all species of the subfamily Murinae that have been examined (including Mus minutoides - this study, Rhabdomys pumilio, Rattus norvegicus and Mus musculus - Bhagwandin et al., 2006); but have not been observed within the cerebral cortex of all other rodent species that have been examined with the AB144P Chemicon/Millipore antibody to choline acetyltransferase (including Tatera brantsii, Cryptomys hottentotus natalensis, Thryonomys swinderianus - Bhagwandin et al., 2006; Hystrix africaeaustralis Limacher et al., 2008; Bathyergus suillus, Cryptomys hottentotus pretoriae, Bhagwandin et al., 2008). In studies of the occurrence of cholinergic neurons in the cerebral cortex of $R$. norvegicus, it was observed that up to $80 \%$ of these cortical cholinergic neurons were also 
immunoreactive for vasoactive intestinal polypeptide (VIP) (Eckenstien and Baughman, 1984), and that virtually all of these VIP neurons co-contain gamma-aminobutyric acid (GABA), with $88 \%$ of cortical cholinergic neurons co-containing GABA (Bayraktart et al., 1997). Interesting, no cortical cholinergic neurons were immunoreactive for vesicular acetylcholine transporter (VChAT) (Bhagwandin et al., 2006), indicating that they do not form part of the traditionally defined cholinergic system (Woolf, 1991). The production of acetylcholine by cortical inhibitory interneurons appears to be limited to the rodent subfamily Murinae, and this may be related to increased functional demands on these cortical neurons allowing them to play a modulatory role in cortical microcirculation (Bhagwandin et al., 2006). As to exactly why this would appear only within the subfamily Murinae (cortical cholinergic neurons are not found in the closely related gerbil Tatera brantsii) is unclear at present; however, it is clear that the Murinae are perhaps the most diverse and speciose (over 500 species) of the rodent subfamilies (Jansa and Weksler, 2004), indicating that either potentially some survival advantage is provided by this feature, or that this feature is evolutionarily neutral in terms of survival.

\subsection{The apperance of the locus coeruleus (A6) across rodent species}

The second feature observed in the current study that seems to be limited in its phylogenetic occurrence to the subfamily Murinae, is the appearance of the locus coeruleus, or A6, cluster of noradrenergic neurons. This cluster of neurons, first described by Dahlström and Fuxe (1964) in R. norvegicus, was described as a tightly packed cluster of neurons stretching the majority of the way across the periventricular grey matter from the pontine tegmental border to the floor of the fourth ventricle (Dahlström and Fuxe, 1964; Hökfelt et al., 1976, 1984; Björklund and Lindvall, 1984). Similar descriptions have been furnished for M. musculus (Ginovart et al., 1996; von Coelln et al., 2004) and in the current study for $M$. minutoides. Interestingly, this compact appearance of the neurons forming the locus coeruleus does not occur in the closely related T. brantsii (Moon et al., 2007), or the more distantly related T. swinderianus (Dwarika et al., 2008), H. africaeaustralis (Limacher et al., 2008) or B. suillus and C. hottentotus (Bhagwandin et al., 2008). In these other rodent species a far less compact packing of the noradrenergic neurons forming the locus coeruleus is observed, and in general they do not approach the floor of the fourth ventricle as closely as they do in the Murinae. Again, it is unclear whether any advantage is to be gained by such a change to the structure of the locus coeruleus in the Murinae rodents, but as indicated above, 
the speciose nature of this subfamily does indicate that if anything, the genetic changes associated with this different adult phenotype are certainly not disadvantageous.

\subsection{Systems level brain evolution}

Manger (2005) proposed that all species within an order will have the same organisation of nuclear systems regardless of life history, adult phenotypic variation (including variance in brain size) and time since evolutionary divergence. This concept is supported by the current findings for the African pygmy mouse, which, despite its very small absolute brain mass ( $\sim 275 \mathrm{mg})$, shares the same number and complement of nuclei in the cholinergic, catecholaminergic, serotonergic and orexinergic systems as all other rodent species in which these systems have been studied to date. We can therefore conclude that decreases in absolute brain mass do not necessarily lead to a decrease in the nuclear differentiation of the systems investigated in species belonging to the same order, a finding that has been confirmed across several mammalian orders (Kruger et al., 2010b; Dell et al., 2010). Thus, in the Rodentia, species covering a range of brain masses, phylogenetic relatedness and phenotypes have been examined. The only potential variance regarding brain evolution that has yet to be explored is to examine rodents with a relatively large brain. Two closely related families of rodents, the scaly-tailed squirrels (Anomaluridae) and Springhares (Pedetidae) (Blanga-Kanfi et al., 2009), have relatively large brain sizes and thus would be of interest to examine to evaluate whether organizational complexity is altered with an increase in relative brain size. If, as seems likely, there is no change in organizational complexity at the systems level, these series of studies will have important ramifications for our understanding of systems level brain evolution.

Despite this well-supported generality regarding systems level complexity and evolution in mammalian brains, there are two specific examples that appear to not be entirely consistent with this generality. As outlined above, the Murinae rodents appears to have two features that distinguish them from the remainder of the Rodentia, the second example being that two species of microchiropterans (Cardioderma cor and Coleura afra) have three features (presence of a parabigeminal nucleus and the dorsal caudal nucleus of the ventral tegmental area, A10dc, and the poor expression of the ventral division of the hypothalamic group, $\mathrm{A} 15 \mathrm{v}$ ) that distinguish them from the remainder of microchiropterans that have been studied (Kruger et al., 2010b). The microchiroptera and the Rodentia, especially the Muroidea, are two extremely speciose mammalian groupings with long evolutionary 
histories. Due to this, and the diversity of the species within these groupings, it is perhaps not surprising that some lineages within these groups have evolved specific apomorphies not represented in the remaining species of the order to which they belong (are they on an evolutionary trajectory that may lead to the establishment of a new mammalian order?). As noted above, the genetic alterations related to the appearance of these specific traits may be neutral in nature (unlike in experimentally manipulated mice, von Coelln et al., 2004), or they may indeed have some role that proves to be advantageous to the lineage in which they have appeared; however, the fact that these apomorphies do appear is of great interest. The laboratory rat and mouse ( $R$. norvegicus and $M$. musculus) are known to be the two species most widely used in the neurosciences (Manger et al., 2008) and both have the Murinae apomorphies. Based on the range of rodents studied to date, this independent evolution of the cortical cholinergic neurons and the compact appearing locus coeruleus in the Murinae is cause for some concern, as it is highly likely that observations regarding these portions of the cholinergic and catecholaminergic systems will be difficult to translate to other species, especially humans that may have independently evolved similar apomorphies. It might also be true that these apomorphies in the different lineages (Murinae and Homo) are analogous, which would also be of great interest. Clearly, further comparative observational and functional assessments are required to determine whether any potential advantages or disadvantages to the extensive use of rats and mice as experimental animal models are apparent.

\section{Acknowledgments}

This work was supported by funding from the South African National Research Foundation (PRM, NCB), SIDA (KF) and by a fellowship with the Postdoc-Programme of the German Academic Exchange Service, DAAD (NP). We thank Prof. Neville Pillay of the University of the Witwatersrand for his kind assistance with obtaining some of the specimens used in this study.

\section{References}

Agnati, L.F., Franzen, O., Ferre, S., Leo, G., Franco, R., Fuxe, K., 2003. Possible role of intramembrane receptor-receptor interactions in memory and learning via formation of long- 
lived heteromeric complexes: focus on motor learning in the basal ganglia. J. Neural. Transm. Suppl. 65, 1-28.

Baldo, B.A., Daniel, R.A., Berridge, C.W., Kelley, A.E., 2003. Overlapping distributions of orexin/hypocretin- and dopamine-beta-hydroxylase immunoreactive fibers in rat brain regions mediating arousal, motivation, and stress. J. Comp. Neurol. 464, 220-237.

Bayraktar, T., Staiger, J.F., Acsady, L., Cozzari, C., Freund, T.F., Zilles, K., 1997. Colocalization of vasoactive intestinal polypeptide, gamma-aminobutyric acid and choline acetyltransferase in the neocortical interneurons of the adult rat. Brain Res. 757, 209-217.

Bhagwandin, A., Fuxe, K., Manger, P.R., 2006. Choline acetyltransferase immunoreactive cortical interneurons do not occur in all rodents: a study of the phylogenetic occurrence of this neural characteristic. J. Chem. Neuroanat. 32, 208-216.

Bhagwandin, A., Fuxe, K., Bennett, N.C., Manger, P.R., 2008. Nuclear organization and morphology of cholinergic, putative catecholaminergic and serotonergic neurons in the brains of two species of African mole-rat. J. Chem. Neuroanat. 35, 371-387.

Bhagwandin, A., Fuxe, K., Bennett, N.C., Manger, P.R., 2011. Distribution of orexinergic neurons and their terminal networks in the brains of two species of African mole rats. J. Chem. Neuroanat. 41, 32-42.

Björklund, A., Lindvall,O., 1984. Dopamine-containing systems in the CNS, in: Björklund, A., Hökfelt, T. (Eds.), Handbook of Chemical Neuroanatomy, vol. 2. Classical Neurotransmitters in the CNS, part 1. Elsevier, Amsterdam, pp. 55-122.

Blanga-Kanfi, S., Miranda, H., Penn, O., Pupko, T., DeBry, R.W., Huchon, D., 2009. Rodent phylogeny revised: analysis of six nuclear genes from all major rodent clades. BMC Evo. Biol. 9, 71.

Chen, C.T., Dun, S.L., Kwok, E.H., Dun, N.J., Chang, J.K., 1999. Orexin A-like immunoreactivity in the rat brain. Neurosci. Lett. 260, 161-164.

Cutler, D.J., Morris, R., Sheridhar, V., Wattam, T.A.K., Kolmes, S., Patel, S., Atch, J.R.S., Wilson, S., Buckingham, R.E., Evans, M.L., Leslie, R.A., Williams, G., 1999. Differential distribution of orexin-A and orexin-b immunoreactivity in the rat brain and spinal cord. Peptides 20, 1455-1470. 
Dahlström, A., Fuxe, K., 1964. Evidence for the existence of monoamine-containing neurons in the central nervous system. I. Demonstration of monoamine in the cell bodies of brainstem neurons. Acta Physiol. Scand. 62, 1-52.

Da Silva, J.N., Fuxe, K., Manger, P.R., 2006. Nuclear parcellation of certain immunohistochemically identifiable neuronal systems in the midbrain and pons of the Highveld mole-rat (Cryptomys hottentotus). J. Chem. Neuroanat. 31, 37-50.

Dell, L-A., Kruger, J-L., Bhagwandin, A., Jillani, N.E., Pettigrew, J.D., Manger, P.R., 2010. Nuclear organization of cholinergic, putative catecholaminergic and serotonergic systems in the brains of two megachiropteran species. J. Chem. Neuroanat. 40, 177-195.

Downs, C.T., Perrin, M.R., 1996. The thermal biology of southern Africa's smallest rodent, Mus minutoides. S. Afr. J. Sci. 92, 282-285.

Dwarika, S., Maseko, B.C., Ihunwo, A.O., Fuxe, K., Manger, P.R., 2008. Distribution and morphology of putative catecholaminergic and serotonergic neurons in the brain of the greater canerat, Thryonomys swinderianus. J. Chem. Neuroanat. 35, 108-122.

Ebbesson, S.O., 1980. The parcellation theory and its relation to interspecific variability in brain organization, evolutionary and ontogenetic development, and neuronal plasticity. Cell Tissue Res. 213, 179-212.

Eckenstein, F., Baughman, R.W., 1984. Two types of cholinergic innervation in the cortex, one co-localised with vasoactive intestinal polypeptide. Nature 309, 153-155.

Espana, R.A., Reis, K.M., Valentino, R.J., Berridge, C.W., 2005. Organization of hypocretin/orexin efferents to locus coeruleus and basal forebrain arousal-related structures. J. Comp. Neurol. 481, 160-178.

Feldman, J.L., Ellenberger, H.H., 1988. Central coordination of respiratory and cardiovascular control in mammals. Annu. Rev. Physiol. 50, 593-606.

Ferguson, A.V., Samson, W.K., 2003. The orexin/hypocretin system: a critical regulator of neuroendocrine and autonomic function. Front. Neuroendocrin. 24, 141-150.

Ferreira, G., Meurisse, M., Tillet, Y., Levy, F., 2001. Distribution and co-localization of choline acetyltransferase and P75 neurotrophin receptors in the sheep basal forebrain: implications for the use of a specific cholinergic immunotoxin. Neuroscience. 104, 419-439. 
Fuxe, K., 1964. Cellular localization of monoamines in the median eminence and the infundibular stem of some mammals. Zeitschrift für Zellforschung 61, 710-724.

Fuxe, K., Hökfelt, T., Ungerstedt, U., 1969. Distribution of monoamines in the mammalian central nervous system by histochemical studies, in: Hooper, G. (Ed.), Metabolism of Amines in the Brain. Macmillan, London, pp. 10-22.

Fuxe, K., Hökfelt, T., Ungerstedt, U., 1970. Morphological and functional aspects of central monoamine neurons. Int. Rev. Neurobiol. 13, 93-126.

Fuxe, K., Härfstrand, A., Agnati, L.F., Kalia, M., Fredholm, B., Svensson, T., Gustafsson, J.A., Lang, R., Ganten, D., 1987. Central catecholamine-neuropeptide Y interactions at the pre- and postsynaptic level in cardiovascular centers. J. Cardiovasc. Pharmacol. 10, Suppl 12, S1-13.

Gallyas, F., 1979. Silver staining of myelin by means of physical development. Neurolog. Res. 1, 203-209.

Gravett, N., Bhagwandin, A., Fuxe, K., Manger, P.R., 2011. Distribution of orexin-A immunoreactive neurons and their terminal networks in the brain of the rock hyrax, Procavia capensis. J. Chem. Neuroanat. 41, 86-96.

Ginovart, N., Marcel, D., Bezin, L., Garcia, C., Gagne, C., Pujol, J.F., Weissman, D., 1996. Tyrosine hydroxylase expression within Balb/C and C57Black6 mouse locus coeruleus. I. Topological organization and phenotypic plasticity of the enzyme-containing cell population. Brain Res. 721, 11-21.

Hökfelt, T., Johansson, O., Fuxe, K., Goldstein, M., Park, D., 1976. Immunohistochemical studies on the localization and distribution of monoamine neuron systems in the rat brain. I. Tyrosine hydroxylase in the mes- and diencephalon. Med. Biol. 54, 427-53.

Hökfelt, T., Martenson, R., Björklund, A., Kleinau, S., Goldstein, M., 1984. Distributional maps of tyrosine-hydroxylase-immunoreactive neurons in the rat brain, in: Björklund, A., Hökfelt, T. (Eds.), Handbook of Chemical Neuroanatomy, vol. 2. Classical Neurotransmitters in the CNS, part 1. Elsevier, Amsterdam, pp. 277-379.

Jacobs, B.L., Azmitia, E.C., 1992. Structure and function of the brain serotonin system. Physiol. Rev. 72, 165-229. 
Jansa, S.A., Weksler, M., 2004. Phylogeny of the murid rodents: relationships within and among major lineages as determined by IRBP gene sequences. Mol. Phylogenet. Evol. 31, $256-276$.

Jones, B.E., Cuello, A.C., 1989. Afferents to the basal forebrain cholinergic cell area from pontomesencephalic catecholamine, serotonin and acetylcholine neurons. Neuroscience. 31 , $37-61$.

Kerley, G.I.H., 1991. Seed removal by rodents, birds and ants in the semiarid Karoo, South Africa. J. Arid. Environ. 20, 63-69.

Khorooshi, R.M., Klingenspor, M., 2005. Neuronal distribution of melanin-concentrating hormone, cocaine- and amphetamine regulated transcript and orexin B in the brain of the Djungarian hamster (Phodopus sungorus). J. Chem. Neuroanat. 29, 137-148.

Kitahama, K., Geffard, M., Okamura, H., Nagatsu, I., Mons, N., Jouvet, M., 1990.

Dopamine- and dopa-immunoreactive neurons in the cat forebrain with reference to tyrosine hydroxylase-immunohistochemistry. Brain Res. 518, 83-94.

Kitahama, K., Sakamoto, N., Jouvet, A., Nagatsu, I., Pearson, J., 1996. Dopamine-betahydroxylase and tyrosine hydroxylase immunoreactive neurons in the human brainstem. J. Chem. Neuroanat. 10, 137-146.

Kirouac, G.J., Parson, M.P., Li, S., 2005. Orexin (hypocretin) innervation of the paraventricular nucleus of the thalamus. Brain Res. 1059, 179-188.

Kruger, J-L., Dell, L-A., Pettigrew, J.D., Manger, P.R., 2010a. Cellular location and major terminal networks of the orexinergic system in the brains of five microchiropteran species. J. Chem. Neuroanat. 40, 256-262.

Kruger, J-L., Dell, L-A., Bhagwandin, A., Jillani, N.E.,Pettigrew, J.D., Manger, P.R., 2010 b. Nuclear organization of cholinergic, putative catecholaminergic and serotonergic systems in the brains of five microchiropteran species. J. Chem. Neuroanat. 40, 210-222.

Leshin, L.S., Kraeling, R.R., Kineman, R.D., Barb, C.R., Rampacek, G.B., 1995. Immunocytochemical distribution of catecholamine-synthesizing neurons in the hypothalamus and pituitary gland of pigs: tyrosine hydroxylase and dopamine- $\beta$-hydroxylase. J. Comp. Neurol. 364, 151-168. 
Limacher, A.M., Bhagwandin, A., Fuxe, K., Manger, P.R., 2008. Nuclear organization and morphology of cholinergic, putative catecholaminergic and serotonergic neurons in the brain of the Cape porcupine (Hystrix africaeaustralis): Increased brain size does not lead to increased organizational complexity. J. Chem. Neuroanat. 36, 33-52.

Manger, P.R., 2005. Establishing order at the systems level in mammalian brain evolution. Brain Res. Bull. 66, 282-289.

Manger, P.R., Cort, J., Ebrahim, N., Goodman, A., Henning, J., Karolia, M., Rodrigues, S.L., Strkalj, G., 2008. Is $21^{\text {st }}$ Century neuroscience too focussed on the rat/mouse model of brain function and dysfunction? Front. Neuroanat. 2, 5.

Maseko, B.C., Bourne, J.A., Manger, P.R., 2007. Distribution and morphology of cholinergic, putative catecholaminergic and serotonergic neurons in the brain of the Egyptian Rousette flying fox, Rousettus aegyptiacus. J. Chem. Neuroanat. 34, 108-127.

McGranaghan, P.A., Piggins, H.D., 2001. Orexin A-like immunoreactivity in the hypothalamus and thalamus of the Syrian hamster (Mesocricetus auratus) and Siberain hamster (Phodopus sungorus), with special reference to circadian structures. Brain Res. 904, 234-244.

Meister, B., Hökfelt, T., Steinbusch, H.W., Skagerberg, G., Lindvall, O., Geffard, M., Joh, T.H., Cuello, A.C., Goldstein, M., 1988. Do tyrosine hydroxylase-immunoreactive neurons in the ventrolateral arcuate nucleus produce dopamine or only L-dopa? J. Chem. Neuroanat. 1, 59-64.

Mintz, E.M., van Den Pol, A.N., Casano, A.A., Albers, H.E., 2001. Distribution of hypocretin (orexin) immunoreactivity in the central nervous system of Syrian hamsters (Mesocricetus auratus). J. Chem. Neuroanat. 21, 225-238.

Monadjem, A., 1999. Populations dynamics of Mus minutoides and Steatomys pratensis (Muridae: Rodentia) in a subtropical grassland in Swaziland. Afr. J. Ecol. 37, 202-210.

Moon, D.J., Maseko, B.C., Ihunwo, A., Fuxe, K., Manger, P.R., 2007. Distribution and morphology of catecholaminergic and serotonergic neurons in the brain of the highveld gerbil, Tatera brantsii. J. Chem. Neuroanat. 34, 134-144. 
Nixon, J.P., Smale, L., 2007. A comparative analysis of the distribution of immunoreactive orexin A and B in the brain of nocturnal and diurnal rodents. Behav. Brain Funct. 3, 28.

Novak, C.M., Albers, H.E., 2002. Localization of hypocretin-like immunoreactivity in the brain of the diurnal rodent, Arvicanthis niloticus. J. Chem. Neuroanat. 23, 49-58.

Olson, L., Fuxe, K., 1972. Further mapping out of the central noradrenaline neurons systems: projections of the "subcoeruleus" area. Brain Res. 43, 289-295.

Paxinos, G., Watson, C., 2009. Chemoarchitectonic Atlas of the Mouse Brain. Academic Press, New York.

Peyron, C., Tighe, D.K., van den Pol, A.N., de Lecea, L., Heller, H.C., Sutcliffe, J.G., Kilduff, T.S., 1998. Neurons containing hypocretin (orexin) project to multiple neuronal systems. J. Neurosci. 18, 9996-10015.

Previc, F.H., 1999. Dopamine and the origins of human intelligence. Brain Cogn. 41, 299350.

Reiner, P.B., Fibiger, H.C., 1995. Functional heterogeneity of central cholinergic systems, in: Bloom, F.E., Kupfer, D.J. (Eds.), Psychopharmacology: The Fourth Generation of Progress. Raven, New York, pp 147-153.

Rodrigues, S.L., Maseko, B.C., Ihunwo, A.O., Fuxe, K., Manger, P.R., 2008. Nuclear organization and morphology of serotonergic neurons in the brain of the Nile crocodile, Crocodylus niloticus. J. Chem. Neuroanat. 35, 133-145.

Ruggiero, D.A., Anwar, M., Gootman, P.M., 1992. Presumptive adrenergic neurons containing phenylethanolamine $\mathrm{N}$-methyltransferase immunoreactivity in the medulla oblongata of neonatal swine. Brain Res. 583, 105-119.

Siegel, J.M., 2006. The stuff dreams are made of: anatomical substrates of REM sleep. Nat. Neurosci. 9, 721-722.

Skagerberg, G., Meister, B., Hökfelt, T., Lindvall, O., Goldstein, M., Joh, T., Cuello, A.C., 1988. Studies on dopamine-, tyrosine hydroxylase- and aromatic L-amino acid decarboxylase-containing cells in the rat diencephalon: comparison between formaldehydeinduced histofluorescence and immunofluorescence. Neuroscience. 24, 605-620. 
Smeets, W.J.A.J., González, A., 2000. Catecholamine systems in the brain of vertebrates: new perspectives through a comparative approach. Brain Res. Rev. 33, 308-379.

Steinbusch, H.W.M., 1981. Distribution of serotonin-immunoreactivity in the central nervous system of the rat - cell bodies and terminals. Neuroscience. 6, 557-618.

Stephan, H., Frahm, H., Baron, G., 1981. New and revised data on volumes of brain structures in insectivores and primates, Folia Primatol. 35, 1-29.

Takakusaki, K., Takahashi, K., Saitoh, K., Harada, H., Okumura, T., Kayama, Y., Koyama, Y., 2005. Orexinergic projections to the cat midbrain mediate alternation of emotional behavioural states from locomotion to cataplexy. J. Physiol. 568, 1003-1020.

Tillet, Y., Kitahama, K., 1998. Distribution of central catecholaminergic neurons: a comparison between ungulates, humans and other species. Histol. Histopathol. 13, $1163-$ 1177.

Törk, I., 1990. Anatomy of the serotonergic system. Ann. N.Y. Acad. Sci. 600, 9-35.

Veyrunes, F., Catalan, J., Tatard, C., Cellier-Holzem, E., Watson, J., Chevret, P., Robinson, T.J., Britton-Davidian, J., 2010. Mitochondrial and chromosomal insights into karyotypic evolution of the pygmy mouse, Mus minutoides, in South Africa. Chromosome Res. 18, 563 574.

Vidal, L., Blanchard, J., Morin, L.P., 2005. Hypothalamic and zona incerta neurons expressing hypocretin, but not melanin concentrating hormone, project to the hamster intergeniculate leaflet. Neuroscience. 134, 1081-1090.

von Coelln, R., Thomas, B., Savitt, J.M., Lim, K.L., Sasaki, M., Hess, E.J., Dawson, V.L., Dawson, T.M., 2004. Loss of locus coeruleus neurons and reduced startle in parkin null mice. Proc. Natl. Acad. Sci. U.S.A. 101, 10744-10749.

Wagner, D., Salin-Pascual, R., Greco, M.A., Shiromani, P.J., 2000. Distribution of hypocretin-containing neurons in the lateral hypothalamus and c-fos-immunoreactive neurons in the VLPO. Sleep Res. Online 3, 35-42.

Wilson, D.E., Reeder, D.M., 2005. Mammal Species of the World: A Taxonomic and Geographic Reference. Johns Hopkins University Press, Baltimore, USA. 
Woolf, N.J., 1991. Cholinergic systems in mammalian brain and spinal cord. Prog.

Neurobiol. 37, 475-524.

Woolf, N.J., Hameroff, S.R., 2001. A quantum approach to visual consciousness. Trends. Cogn. Sci. 5, 472-748.

Zeitzer, J.M., Buckmaster, C.L., Parker, K.J., Hauck, C.M., Lyons, D.M., Mignot, E., 2003. Circadian and homeostatic regulation of hypocretin in a primate model: implications for the consolidation of wakefulness. J. Neurosci. 23, 3555-3560. 


\section{Figure Legends}

Figure 1: Serial drawings of coronal sectiosn through one half of the African pygmy mouse brain from the olfactory bulb through to the spinomedullary junction. $\mathbf{A}$ is the most rostral section, $\mathbf{R}$ the most caudal. The outlines of the architectonic regions were drawn using Nissl and myelin stains and immunoreactive cells marked on the drawings. Solid black circles depict cholinergic neurons, solid triangles depict catecholaminergic neurons (those immunoreactive for tyrosine hydroxylase), open squares depict serotonergic neurons and closed stars represent orexinergic neurons. Each circle, triangle, square or start represents an individual neuron. The figures are approximately $600 \mathrm{~mm}$ apart. See list for abbreviations.

Figure 2: Photomicrographs showing neuronal groups immunoreactive for choline acetyltransferase in the brain of the African pygmy mouse. A. Basal forebrain region showing the diagonal band of Broca (Diag.B), the caudate nucleus $(\mathbf{C})$, nucleus accumbens (N.Acc) and the olfactory tubercle (TOL). Scale bar in $\mathbf{A}=1 \mathrm{~mm}$. ac - anterior commissure; LOT lateral olfactory tract. B. Cerebral neocortex showing the widespread distribution of cortical cholinergic neurons. Inset: high power photomicrographs of the cell bodies of cortical cholinergic neurons. Scale bar of Inset $=50 \mu \mathrm{m}$. C. The dorsal hypothalamic cholinergic nucleus (Hyp.d) lying between and just above the third ventricle (3V) and the fornix (f). GP - globus pallidus; N.Bas - nucleus basalis. D. The medial habenular nucleus (Hbm) and fasciculus retroflexus (fr). E. The pedunculopontine (PPT) and parabigeminal (PBg) nuclei found in the pontine region. F. The laterodorsal tegmental nucleus (LDT) and the pedunculopontine nucleus (PPT) lying immediately beneath the superior cerebellar peduncle (scp). In all images, except $\mathbf{D}$ which is at the midline, medial is to the left, and dorsal to the top. Scale bar in $\mathbf{F}=500 \mu \mathrm{m}$, and applies to $\mathbf{B}-\mathbf{F}$, except for the Inset in $\mathbf{B}$.

Figure 3: Photomicrographs showing neuronal groups that are immunopositive for tyrosine hydroxylase in the brain of the African pygmy mouse. A. In the rostral hypothalamus the dorsal division of the anterior hypothalamic group (A15d) and the rostral periventricular nucleus (A14) were observed. B. In the caudal hypothalamus, the rostral periventricular nucleus (A14), zona incerta cell group (A13) and the tuberal cell group (A12) were observed. OT - optic tract. C. In the ventral midbrain, the dorsal division of the ventral tegmental area (A10d), the retrorubral nucleus $(\mathbf{A 8})$ and the pars reticulata of the substantia nigra (A9v) nuclei were seen. IP - interpeduncular nucleus. D. The compact nature of the locus coeruleus 
(A6c) in the African pygmy mouse is typical of Murid rodents. $4 \mathrm{~V}$ - fourth ventricle; scp superior cerebellar peduncle. In all images, except $\mathbf{A}$ which is at the midline, medial is to the left, and dorsal to the top. Scale bar in $\mathbf{D}=500 \mu \mathrm{m}$, and applies to all.

Figure 4: Photomicrographs showing neuronal groups that are immunopositive for serotonin in the brain of the African pygmy mouse. A. In the ventral portion of the rostral midbrain, the most rostral serotonergic nuclei, the caudal linear nucleus $(\mathbf{C L i})$ and the supralemniscal supralemniscal serotonergic nucleus (B9) where observed above and lateral to the interpeduncular nucleus (IP). VPO - ventral pontine nucleus. B. In the central grey matter of the midbrain the dorsal (DRd), ventral (DRv), interfascicular (DRif) and periperhal (DRp) subdivisions of the dorsal raphe were observed. ca - cerebral aqueduct. C. In the caudal most portion of the dorsal midbrain where the cerebral aqueduct opens in the fourth ventricle (4V) the caudal divison of the dorsal raphe (DRc) and the median raphe nucleus (MnR) were observed. D. In the ventral portions of the rostral medulla, the raphe magnus (RMg), raphe pallidus (RPa) and rostral ventrolateral serotonergic group (RVL) were observed close to the pyramidal tract (py). In all images, except $\mathbf{C}$ which is at the midline, medial is to the left, and dorsal to the top. Scale bar in $\mathbf{D}=500 \mu \mathrm{m}$, and applies to all.

Figure 5: Photomicrograph showing hypothalamic neuronal groups that are immunoreactive for orexine in the brain of the African pygmy mouse. $\mathrm{f}$ - fornix; $\mathrm{Mc}$ - main orexinergic cluster; OT - optic tract; Otc - optic tract orexinergic cluster; Zic - zona incerta orexinergic cluster. Dorsal is to the top and the midline is to the left. Scale bar $=250 \mu \mathrm{m}$. 
B

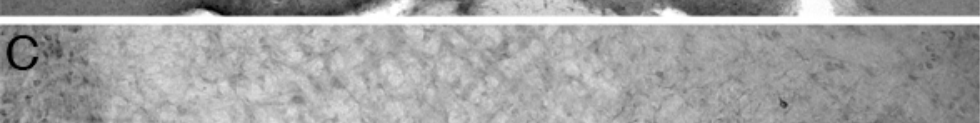
A10d
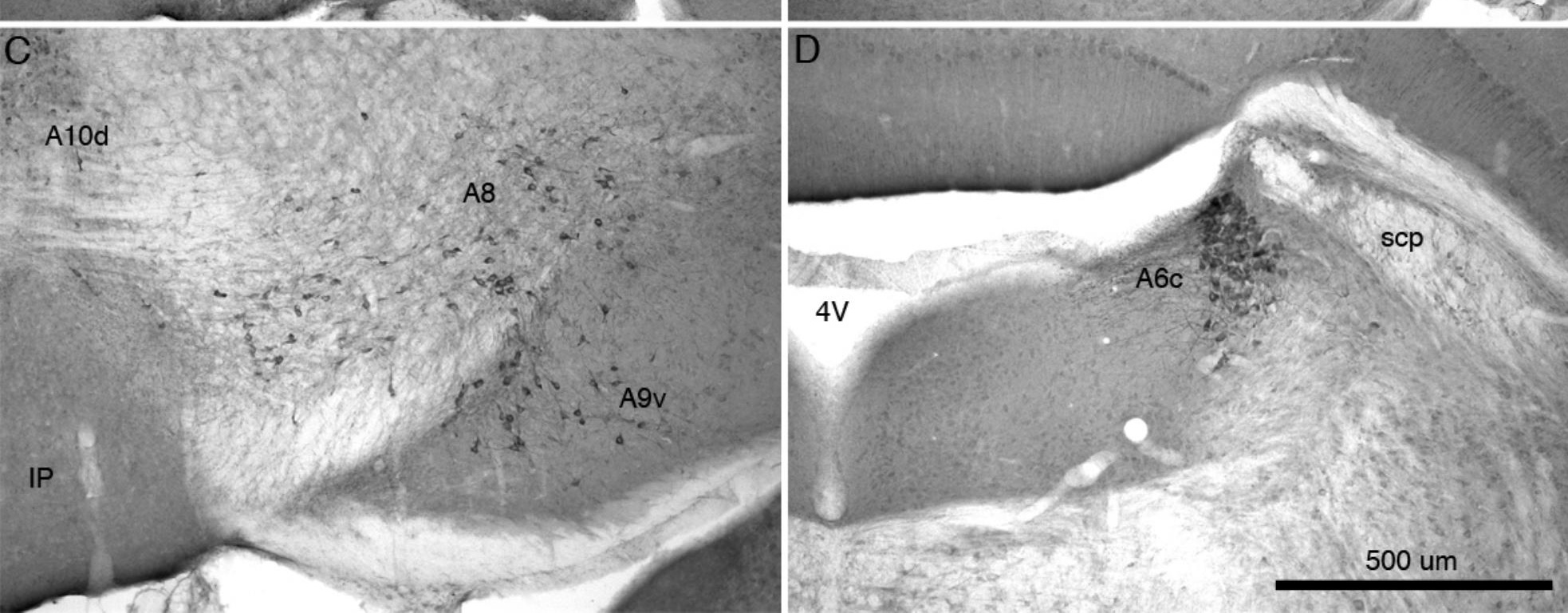


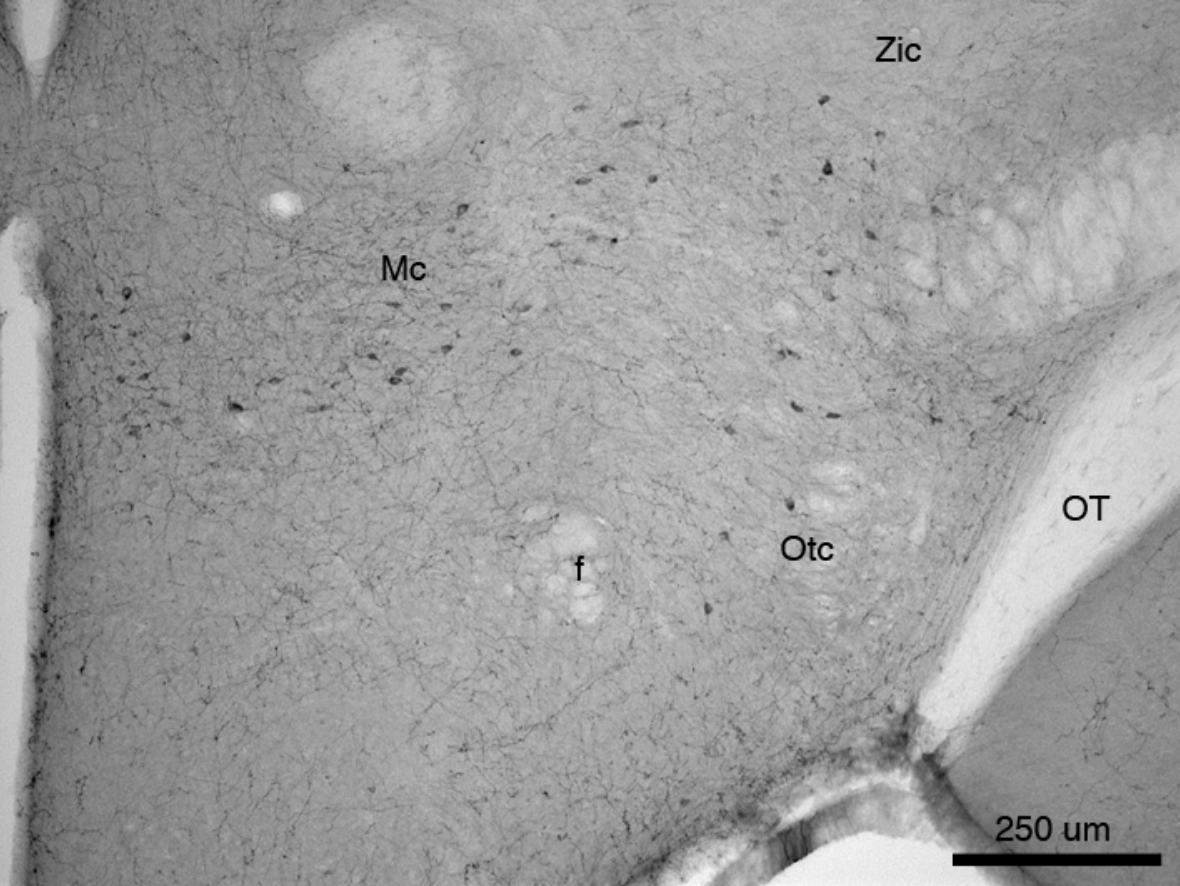

